\title{
A POISSON RELATION FOR CONIC MANIFOLDS
}

\author{
JARED WUNSCH
}

\begin{abstract}
Let $X$ be a compact Riemannian manifold with conic singularities, i.e. a Riemannian manifold whose metric has a conic degeneracy at the boundary. Let $\Delta$ be the Friedrichs extension of the Laplace-Beltrami operator on $X$. There are two natural ways to define geodesics passing through the boundary: as "diffractive" geodesics which may emanate from $\partial X$ in any direction, or as "geometric" geodesics which must enter and leave $\partial X$ at points which are connected by a geodesic of length $\pi$ in $\partial X$. Let DIFF $=\{0\} \cup\{ \pm$ lengths of closed diffractive geodesics $\}$ and GEOM $=\{0\} \cup\{ \pm$ lengths of closed geometric geodesics $\}$. We show that$$
\operatorname{Tr} \cos t \sqrt{\Delta} \in \mathcal{C}^{-n-0}(\mathbb{R}) \cap \mathcal{C}^{-1-0}(\mathbb{R} \backslash \text { GEOM }) \cap \mathcal{C}^{\infty}(\mathbb{R} \backslash \text { DIFF }) .
$$

This generalizes a classical result of Chazarain and Duistermaat-Guillemin on boundaryless manifolds, which in turn follows from Poisson summation in the case $X=S^{1}$.

\section{Introduction}

Let $(X, g)$ be a conic manifold, that is to say, a manifold $X$ with boundary, endowed with a Riemannian metric that takes the form

$$
g=d x^{2}+x^{2} h
$$

in a neighborhood of $\partial X$, with $x$ a boundary defining function and

$$
h \in \mathcal{C}^{\infty}\left(X ; \operatorname{Sym}^{2} T^{*} X\right)
$$

nondegenerate in the sense that $h_{0} \equiv h \uparrow_{x=0}$ is a metric. Trivial examples of conic metrics are obtained by blowing up isolated points on a Riemannian manifold. Less trivial examples include the product cones $\overline{\mathbb{R}_{+}} \times Y$ endowed with metric $d x^{2}+x^{2} h(y, d y)$ with $h$ a metric on $Y$. A conic manifold in the sense defined here can be regarded as the desingularization, or blow-up, of a manifold with conic singularities. The degeneracy of the metric at the boundary makes each boundary component look geometrically like a separate cone point. One geometric consequence of the degeneracy of the metric is the most geodesics fail to strike $\partial X$; indeed through any point close to $\partial X$ there will be precisely one direction in which the exponential map will reach the boundary in short time (see $[7])$.

Let $\Delta$ be the Friedrichs extension of the (nonnegative) Laplace-Beltrami operator on $X$. The propagation of singularities for solutions of the wave equation

$$
\left(D_{t}^{2}-\Delta\right) u=0
$$

Received March 19, 2002. 


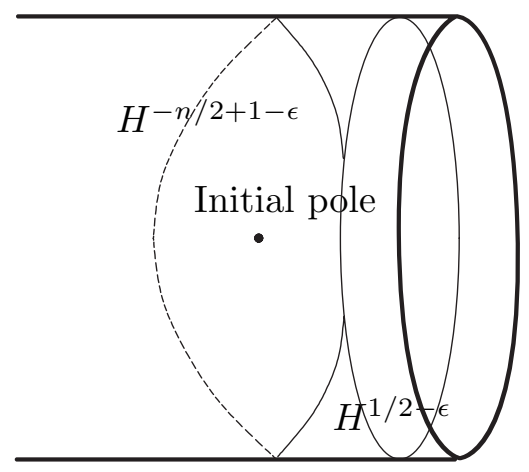

FiguRE 1. The wavefronts of the fundamental solution $\sin t \sqrt{\Delta} / \sqrt{\Delta}$

on conic manifolds was studied by the author and R. Melrose in [7]. The results of that paper showed that while singularities propagate along geodesics over $X^{\circ}$ as described by Hörmander's theorem [6], a singularity striking one component of $\partial X$ will, in general, give rise to a "diffracted wave" emanating spherically from that component of the boundary. The singularity of the diffracted wave, measured away from points which are "geometrically related" to the incoming singularity by time- $\pi$ geodesic flow in $\partial X$, will in many cases be weaker than the incident singularity; for this difference in regularity to manifest itself, it suffices that the incident singularity be not too directly focused upon the boundary, in a sense made precise in [7]. A plausible explanation for this distinction is as follows: If we take a sequence of geodesics in the interior of $X$ passing arbitrarily close, in the limit, to $\partial X$, then there exists a subsequence approaching a continuous path consisting of: a geodesic striking the boundary; followed by a geodesic within the boundary of length $\pi$; followed by a geodesic emanating from the boundary. This outgoing geodesic is thus geometrically related in the sense described above to the incoming one. Thus strong singularities propagate through the boundary as one would expect from their limiting behavior in the interior; the (often weaker) diffractive wave, by contrast, sends singularities out from the boundary along rays which are geometrically inacessible and lie in this sense in a shadow region.

In the crucial example of the fundamental solution $\sin t \sqrt{\Delta} / \sqrt{\Delta}$ with initial pole chosen sufficiently close to the boundary, the diffracted wave is $(n-1) / 2-\epsilon$ derivatives smoother than the main singularity (for all $\epsilon>0$ ), and is furthermore conormal to the radial surface emanating from $\partial X$. Figure 1 shows the main front and the diffractive wave for such a solution, labeled by their Sobolev regularity. In this case the diffractive region is the part of the spherical wave emanating from the boundary that does not lie in the closure of the main front.

In the case of product cones, these propagation results follow from the explicit construction of the fundamental solution carried out by Cheeger and Taylor $[3,2]$ See also the work of Gerard-Lebeau [5] for an analogous result in analytic category in the setting of manifolds with boundary. 
Let $X$ be a conic manifold and let $Y_{i}$ denote the boundary components of $X$, with $i=1, \ldots, K$. Based on the two different kinds of propagation of singularities described above, we now make two different definitions of geodesics passing through $\partial X$.

Definition 1. A diffractive geodesic on $X$ is a union of a finite number of closed, oriented geodesic segments $\gamma_{1}, \ldots, \gamma_{N}$ in $X$ such that all end points except possibly the initial point in $\gamma_{1}$ and the final point of $\gamma_{N}$ lie in $\partial X$, and $\gamma_{i}$ ends at the same boundary component at which $\gamma_{i+1}$ begins, for $i=1, \ldots, N-1$.

A geometric geodesic is a diffractive geodesic such that in addition, the final point of $\gamma_{i}$ and the initial point of $\gamma_{i+1}$ are connected by a geodesic of length $\pi$ in $\partial X$ (w.r.t. the metric $h_{0}=h \uparrow_{\partial X}$ ) for $i=1, \ldots, N-1$.

A diffractive geodesic is closed if the initial point of $\gamma_{1}$ and the final point of $\gamma_{N}$ coincide in $X^{\circ}$ and if $\gamma_{1}, \gamma_{N}$ have the same tangent there, or if the initial point of $\gamma_{1}$ and the final point of $\gamma_{N}$ lie in the same component of $\partial X$.

A geometric geodesic is closed if the initial point of $\gamma_{1}$ and the final point of $\gamma_{N}$ coincide in $X^{\circ}$ and if $\gamma_{1}, \gamma_{N}$ have the same tangent there, or if the initial point of $\gamma_{1}$ and the final point of $\gamma_{N}$ lie in $\partial X$ and are connected by a geodesic of length $\pi$ in $\partial X$.

The length of a diffractive geodesic is the sum of the lengths of its segments $\gamma_{i}$.

Let

$$
\mathrm{DIFF}=\{ \pm \text { lengths of closed diffractive geodesics }\} \cup\{0\}
$$

and

$$
\text { GEOM }=\{ \pm \text { lengths of closed geometric geodesics }\} \cup\{0\} .
$$

Let $k \in \mathbb{N}$. Let $u$ be a distribution on $\mathbb{R}$. We write $u \in \mathcal{C}^{-k-0}(\mathbb{R})$ if $D_{t}^{-k-\delta} \phi u \in L_{\mathrm{loc}}^{\infty}(\mathbb{R})$ for all $\delta>0$ and $\phi \in \mathcal{C}_{c}^{\infty}(\mathbb{R})$. Note that if $u$ has compact support, this coincides with $u \in C_{*}^{-k-\delta}(\mathbb{R})$ for all $\delta>0$ in the notation of [10], Section 13.8 .

This paper is devoted to establishing the following "Poisson relation" (socalled because it generalized part of the Poisson summation formula from the case in which $X$ is the boundaryless manifold $S^{1}$ ):

Theorem. Let $\Delta$ be the Friedrichs extension of the Laplacian on a compact conic manifold. Then

$$
\operatorname{Tr} \cos t \sqrt{\Delta} \in \mathcal{C}^{-n-0}(\mathbb{R}) \cap \mathcal{C}^{-1-0}(\mathbb{R} \backslash \text { GEOM }) \cap \mathcal{C}^{\infty}(\mathbb{R} \backslash \text { DIFF }) .
$$

This generalizes the classical result on a boundaryless manifold, due to Chazarain [1] and Duistermaat-Guillemin [4], that the trace is smooth away from $\{0\} \cup\{ \pm$ lengths of closed geodesics $\}$. Note that when the closed geodesics on a compact, boundaryless manifold are isolated and nondegenerate, the results of Chazarain and Duistermaat-Guillemin show that $\operatorname{Tr} \cos t \sqrt{\Delta} \in \mathcal{C}^{-1-0}(\mathbb{R} \backslash\{0\})$. It thus seems possible that subject to an appropriate nondegeneracy assumption, regularity in $\mathcal{C}^{-1-0}$ might also hold at lengths of all closed geometric geodesics. Thus it is possible that the strength of the singularities in the wave trace allows 
us to distinguish lengths of geometric geodesics from diffractive ones only when the former are actually degenerate.

It is natural to ask whether a full trace formula of the type proved by Chazarain and Duistermaat-Guillemin, with asymptotic expansions at the singularities, holds on conic manifolds. Unfortunately the tools developed in [7] are insufficiently constructive to demonstrate such an expansion except perhaps at $t=0$. A more accessible future direction would be to extend the results of this paper to give a filtration of the singularities according to how many diffractive interactions with the boundary a closed geodesic undergoes: subject to some nondegeneracy assumptions, more diffractions should result in a weaker singularity.

\section{Geodesic flow}

We begin by describing the lifts of the diffracted and geometric geodesics to (a rescaled version of) the cotangent bundle.

In [7], it is shown that by appropriate choice of product structure $(x, y)$ near a boundary component $Y_{i}$, we may bring any conic metric to the reduced form

$$
g=d x^{2}+x^{2} h(x, y, d y)
$$

i.e. we can reduce $h$ to a smooth family in $x$ of metrics on $Y_{i}$. The existence of this normal form is equivalent to the existence of a fibration of a neighborhood of $Y_{i}$ by short geodesics reaching $Y_{i}$ : with the metric in the form (1) the geodesics reaching $Y_{i}$ are precisely those of the form $y=y_{0}, x=x_{0}-t$. Henceforth, we work in such product coordinates.

Let ${ }^{b} T^{*} X$ denote the b-cotangent bundle of $X$, i.e. the dual of the bundle whose sections are smooth vector fields tangent to $\partial X$. Let ${ }^{b} S^{*} X$ denote the corresponding sphere bundle. Let $\xi d x / x+\eta \cdot d y$ denote the canonical one-form on ${ }^{b} T^{*} X$.

Let $K_{g}$ be the Hamilton vector field for $g / 2=\left(\xi^{2}+h(x, y, \eta)\right) /\left(2 x^{2}\right)$, the symbol of $\Delta / 2$ on ${ }^{b} T^{*} X$; note that $K_{g}$ is merely the geodesic spray in ${ }^{b} T^{*} X$ with velocity $\sqrt{g}$. It is convenient to rescale this vector field so that it is both tangent to the boundary of $X$ and homogeneous of degree zero in the fibers. Near a boundary component $Y_{i}$, for a metric in the reduced form (1), we have (see [7])

$$
K_{g}=x^{-2}\left(H_{Y_{i}}(x)+\left(\xi^{2}+h(x, y, \eta)+\frac{x}{2} \frac{\partial h}{\partial x}\right) \partial_{\xi}+\xi x \partial_{x}\right),
$$

where $H_{Y_{i}}(x)$ is the geodesic spray in $Y_{i}$ with respect to the family of metrics $h(x, \cdot)$. Hence the desired rescaling is

$$
Z=\frac{x}{\sqrt{g}} K_{g}
$$

By the homogeneity of $Z$, if we radially compactify the fibers of the cotangent bundle and identify ${ }^{b} S^{*} X$ with the "sphere at infinity" then $Z$ is tangent to 
${ }^{b} S^{*} X$, and may be restricted to it. Henceforth, then, we let $Z$ denote the restriction of $(x / \sqrt{g}) K_{g}$ to the compact manifold ${ }^{b} S^{*} X$ on which the coordinates $\xi, \eta$ have been replaced by the (redundant) coordinates

$$
(\bar{\xi}, \bar{\eta})=\left(\xi / \sqrt{\xi^{2}+h(\eta)}, \eta / \sqrt{\xi^{2}+h(\eta)}\right) .
$$

$Z$ vanishes only at certain points $x=\bar{\eta}=0$ over $\partial X$, hence the closures of maximally extended integral curves of this vector field can only begin and end over $\partial X$. Since $Z$ is tangent to the boundary, such integral curves either lie entirely over $\partial X$ or lie over $\partial X$ only at their limit points. Interior and boundary integral curves can meet only at limit points in $\{x=\bar{\eta}=0\} \subset{ }^{b} S^{*} X$.

It is helpful in studying the integral curves of $Z$ to introduce the following way of measuring their lengths: Let $\gamma$ be an integral curve of $Z$ over $X^{\circ}$. Let $k$ denote a Riemannian metric on ${ }^{b} S^{*} X^{\circ}$ such that $k(Z, Z)=1$. Let

$$
\omega=x k(\cdot, Z) \in \Omega^{1}\left({ }^{b} S^{*} X\right) \text {. }
$$

Then

$$
\int_{\gamma} \omega=\int_{\gamma} x k(d \gamma / d s, Z) d s=\int_{\gamma} \frac{x}{\sqrt{g}} k\left(K_{g}, Z\right) d s=\int_{\gamma} d s=\operatorname{length}(\gamma)
$$

where $s$ parametrizes $\gamma$ as an integral curve of $K_{g} / \sqrt{g}$, the unit speed geodesic flow. With this motivation in mind, we now define, for each $t \in \mathbb{R}_{+}$, two relations in ${ }^{b} S^{*} X$, a "geometric" and a "diffractive" relation. These correspond to the two different possibilities for geodesic flow through the boundary.

Definition 2. Let $p, q \in{ }^{b} S^{*} X$. We write

$$
p \stackrel{G, t}{\sim} q
$$

if there exists a continuous, piecewise smooth curve $\gamma:[0,1] \rightarrow{ }^{b} S^{*} X$ with $\gamma(0)=p, \gamma(1)=q$, such that $[0,1]$ can be decomposed into a finite union of closed subintervals $I_{j}$, intersecting at their endpoints, where

1. on each $I_{j}^{\circ}, \gamma$ is a (reparametrized) positively oriented integral curve of $Z$ in ${ }^{b} S^{*} X$,

2. On successive intervals $I_{j}$ and $I_{j+1}$, interior and boundary curves alternate,

3. $\int_{\gamma} \omega=t$, with $\omega$ as defined in (3).

We write

$$
p \stackrel{D, t}{\sim} q
$$

if there exists a piecewise smooth (not necessarily continuous) curve $\gamma:[0,1] \rightarrow$ ${ }^{b} S^{*} X$ with $\gamma(0)=p, \gamma(1)=q$, such that $[0,1]$ can be decomposed into a finite union of closed subintervals $I_{j}$, intersecting at their endpoints, where

1. on each $I_{j}^{\circ}, \gamma$ is a (reparametrized) positively oriented integral curve of $Z$ in ${ }^{b} S^{*} X^{\circ}$,

2. the final point of $\gamma$ on $I_{j}$ and the initial point of $\gamma$ on $I_{j+1}$ lie over the same component of $\partial X$,

3. $\int_{\gamma} \omega=t$. 
Integral curves of $Z$ over $X^{\circ}$ are lifts of geodesics in $X^{\circ}$, and it follows from (2) that the maximally extended integral curves of $Z$ in ${ }^{b} S_{\partial X}^{*} X$ are lifts of geodesics of length $\pi$ in $\partial X$ (see [7] for details), hence:

Proposition 3. $p \stackrel{G, t}{\sim} q$ iff $p$ and $q$ are connected by a (lifted) geometric geodesic of length $t$.

$p \stackrel{D, t}{\sim} q$ iff $p$ and $q$ are connected by a (lifted) diffractive geodesic of length $t$.

Proposition 4. The sets $\{(p, q, t): p \stackrel{G, t}{\sim} q\}$ and $\{(p, q, t): p \stackrel{D, t}{\sim} q\}$ are closed subsets of ${ }^{b} S^{*} X \times{ }^{b} S^{*} X \times \mathbb{R}_{+}$.

Proof. Let $\gamma_{i}$ be a sequence of "geometric" curves as described in the first part of Definition 2 with initial and final points $p_{i}, q_{i}$ where the $p_{i}$ converge to $p$ and $q_{i}$ to $q$, and with $\int_{\gamma_{i}} \omega \rightarrow t$. Parametrize $\gamma_{i}=\gamma_{i}(s)$ with respect to length in some fixed, nondegenerate Riemannian metric $G$ on ${ }^{b} S^{*} X$. The lengths of $\gamma_{i}$ w.r.t. $G$ are uniformly bounded, since only a finite number of segments are involved (note that the number of smooth integral curves into which $\gamma_{i}$ decomposes is a priori bounded because each interior segment has length bounded below by $\left.\min _{j, k} d_{g}\left(Y_{j}, Y_{k}\right)\right)$. The $\gamma_{i}(s)$ are then equicontinuous, hence applying Ascoli's theorem we may pass to a subsequence that converges uniformly to a path $\gamma(s)$. This must be a geometric curve as in Definition 2, since it is continuous and away from the singular points of $Z$, a limit of integral curves is an integral curve. At the set $\{x=\bar{\eta}=0\} \subset{ }^{b} S_{\partial X}^{*} X$ where $Z$ vanishes, it vanishes nondegenerately (see $\S 1$ of [7]), so we may arrange that the coefficients of the metric $k$ in (3) be bounded by some multiple of $\left(x^{2}+\bar{\eta}^{2}\right)^{-1}$. Since $x Z$ vanishes quadratically at $\{x=\bar{\eta}=0\}, \omega=x k(Z, \cdot)$ is in fact in $L^{\infty}$. Therefore $\int_{\gamma} \omega=\lim \int_{\gamma_{i}} \omega=t$, as desired.

The result for the diffractive relation follows via a similar argument.

As a consequence, we have:

Proposition 5. For any compact conic manifold, DIFF and GEOM are closed subsets of $\mathbb{R}$. For any $T \in \mathbb{R}_{+}$, the set of lifts of closed diffractive resp. geometric geodesics of length $T$ is a compact subset of ${ }^{b} S^{*} X$.

\section{Results from $[7]$}

In [7], the domains of a range of powers of the Friedrichs Laplacian on a conic manifold $X$ are identified in terms of certain Sobolev spaces. We therefore begin by recalling some notions about b-pseudodifferential operators and Sobolev spaces.

Let $\Psi_{\mathrm{b}}^{m}(X)$ denote the space of b-pseudodifferential (or "totally characteristic") operators of order $m$ on $X$, developed by Melrose [8]. This calculus of operators is designed to contain the vector fields tangent to $\partial X$ as operators of order one. The symbol of an operator in the b-calculus naturally lies in the the b-cotangent bundle ${ }^{b} T^{*} X$ introduced above. Let $H_{b}^{m}(X)$ denote the associated 
scale of Sobolev spaces, defined as usual with respect to the measure $d x d y / x$; for positive integral orders, these spaces may be defined by regularity under application of products of vector fields of the form $x D_{x}, D_{y_{i}}$.

Let $(X, g)$ be a conic manifold. Let $L_{g}^{2}(X)$ denote the space of metric squareintegrable functions on $X$ and let $\mathcal{D}_{s}=\operatorname{Dom}\left(\Delta^{s / 2}\right)$, where $\Delta$ is the Friedrichs extension of the conic Laplacian on $X$ acting on $L_{g}^{2}$.

The following is proven in [7]:

Proposition 6. For $s \in(-n / 2, n / 2), \mathcal{D}_{s}=x^{-n / 2+s} H_{b}^{s}(X)$.

(Note that the factor $x^{-n / 2}$ arises because $L_{g}^{2}(X)=x^{-n / 2} L_{\mathrm{b}}^{2}(X)$.)

The Cauchy problem for the wave equation is well-posed on Cauchy data in $\mathcal{D}_{s} \oplus \mathcal{D}_{s-1}$ for any $s \in \mathbb{R}$ and yields a solution $u \in \mathcal{C}^{0}\left(\mathbb{R} ; \mathcal{D}_{s}\right) \cap \mathcal{C}^{1}\left(\mathbb{R} ; \mathcal{D}_{s-1}\right)$. It is convenient to introduce the family of operators $\Theta_{r}, r \in \mathbb{R} \backslash \mathbb{Z}$ defined by

$$
\kappa\left(\Theta_{r}\right)\left(t, t^{\prime}\right)=\psi\left(t-t^{\prime}\right) \kappa\left(\left|D_{t}\right|^{r}\right)\left(t, t^{\prime}\right)
$$

where $\psi(t)$ is a smooth function of compact support, equal to 1 near $t=0$, and $\kappa$ denotes Schwartz kernel. (Hence $\Theta_{r}$ is a version of $\left|D_{t}\right|^{r}$, but with Schwartz kernel cut off to have proper support.) Using the functional calculus, we easily see that $\Theta_{r}$ maps a solution to the conic wave equation $u \in \mathcal{C}^{0}\left(\mathbb{R} ; \mathcal{D}_{s}\right) \cap \mathcal{C}^{1}\left(\mathbb{R} ; \mathcal{D}_{s-1}\right)$ to a solution in $\mathcal{C}^{0}\left(\mathbb{R} ; \mathcal{D}_{s-r}\right) \cap \mathcal{C}^{1}\left(\mathbb{R} ; \mathcal{D}_{s-r-1}\right)$. Moreover, $\Theta_{r} \Theta_{-r} u-u \in \mathcal{C}\left(\mathbb{R} ; \mathcal{D}_{\infty}\right)$. (See [7] for details.)

Let $\mathcal{I}_{1}(H)$ denote the space of trace class operators on the Hilbert space $H$. The following lemma will be crucial in what follows, as it yields a criterion for an operator to be trace-class in terms of its mapping properties on domains.

Lemma 7. If $A: \mathcal{D}_{s} \rightarrow \mathcal{D}_{s+r}$ with $r>n$ then $A \in \mathcal{I}_{1}\left(\mathcal{D}_{s}\right)$.

Proof. To begin with we may reduce to the case in which $s=0$, hence $\mathcal{D}_{s}=L_{g}^{2}$, by using the isomorphism $\langle\Delta\rangle^{s / 2}: \mathcal{D}_{s^{\prime}} \rightarrow \mathcal{D}_{s^{\prime}-s}$.

It follows from results of [7] that $A: x^{n / 2} L_{\mathrm{b}}^{2}(X) \rightarrow x^{-\epsilon} H_{b}^{n+\epsilon}(X)$. Let $B$ be an elliptic element of $\Psi_{\mathrm{b}}^{n+\epsilon}(X)$, and let $C \in \Psi_{\mathrm{b}}^{-n-\epsilon}(X)$ be a "small parametrix" for $B$ as constructed in [9], so that $C B-I=R \in \Psi_{\mathrm{b}}^{-\infty}(X)$. We now write

$$
A=\left(C x^{n / 2-\epsilon}\right)\left(x^{-n / 2+\epsilon} B A\right)+\left(R x^{\epsilon}\right)\left(x^{-\epsilon} A\right) .
$$

Proposition 4.57 of [9] states that if $\alpha>0$ and $\beta<-n$ then $x^{\alpha} \Psi_{\mathrm{b}}^{\beta}(X) \subset$ $\mathcal{I}_{1}\left(L_{g}^{2}\right)$. Hence $C x^{n / 2-\epsilon}$ and $R x^{\epsilon}$ are of trace-class; since $x^{-n / 2+\epsilon} B A$ and $x^{-\epsilon} A$ are bounded on $L_{g}^{2}$, we conclude that $A$ is of trace class.

Finally, we recall from [7] the propagation results which, together with Lemma 7, will enable us to evaluate the regularity of the wave trace. Let

$$
U(t)=\left(\begin{array}{cc}
\cos t \sqrt{\Delta} & \sin t \sqrt{\Delta} / \sqrt{\Delta} \\
-\sqrt{\Delta} \sin t \sqrt{\Delta} & \cos t \sqrt{\Delta}
\end{array}\right)
$$

be the solution operator to the Cauchy problem for the wave equation on $X$. Let $\Psi_{c}^{*}\left(X^{\circ}\right)$ denote the space of pseudodifferential operators on $X^{\circ}$ with compact 
support. Let $L\left(V_{1}, V_{2}\right)$ denote the space of continuous linear maps between Fréchet spaces $V_{i}$. Let

$$
\mathcal{E}_{s}=\mathcal{D}_{s} \oplus \mathcal{D}_{s-1}
$$

denote the "energy space," with $\mathcal{E}_{\infty}=\bigcap_{s \in \mathbb{R}} \mathcal{E}_{s}, E_{-\infty}=\bigcup_{s \in \mathbb{R}} \mathcal{E}_{s}$.

Proposition 8. Let $I \subset \mathbb{R}_{+}$be contained in a small neighborhood of 0 . Let $A_{1}, A_{2} \in \Psi^{0}\left(X^{\circ}\right)$ be supported near $\partial X$, and assume that that there do not exists points $p \in \mathrm{WF}^{\prime} A_{2}, q \in \mathrm{WF}^{\prime} A_{1}$, and $t \in I$ such that $p \stackrel{D, t}{\sim} q$. Then $A_{1} U(t) A_{2} \in$ $\mathcal{C}\left(I ; L\left(\mathcal{E}_{-\infty}, \mathcal{E}_{\infty}\right)\right)$.

In the following proposition, $x$ and $x^{\prime}$ will denote the boundary defining function (in the product-type coordinates introduced in \$2) in the left and right factors of the Schwartz kernel.

Proposition 9. Let $I, A_{1}, A_{2}$ be as in Proposition 8, and assume that that there do not exists points $p \in \mathrm{WF}^{\prime} A_{2}, q \in \mathrm{WF}^{\prime} A_{1}$, and $t \in I$ such that $p \stackrel{G, t}{\sim} q$. Then for $t \in I$,

$$
A_{1} U(t) A_{2}=\left(\begin{array}{cc}
u_{1} & u_{2} \\
-u_{2} & u_{1}
\end{array}\right)
$$

where $u_{1}$ and $u_{2}$ are conormal distributions at $t=x+x^{\prime}$ in $H^{-1 / 2-\epsilon}$ resp. $H^{1 / 2-\epsilon}$ for all $\epsilon>0$.

A consequence of this proposition, which we will use below, is that subject to the hypotheses, the diagonal term $u_{1}$ of $A_{1} U(t) A_{2}$ is a Fourier integral operator of order $-\epsilon$ in the variable $x$, with values in $\Psi^{-\infty}(Y)$, uniformly in $t$. (Recall that the fundamental solution discussed in the introduction is the off-diagonal term.)

We will also need a version of Proposition 8 which applies up to the boundary of $X$.

Proposition 10. Proposition 8 still holds if either or both of $A_{1}, A_{2}$ are replaced by the operator of multiplication by a smooth function $\psi_{i}$ supported in a neighborhood of a component of $\partial X$, and $\mathrm{WF}^{\prime} A_{i}$ correspondingly replaced by $\pi^{-1}\left(\operatorname{supp} \psi_{i}\right)$.

\section{Proof of the theorem}

As the proof has several parts, with ramifying subcases, this section is similarly subdivided. We begin with a sketch of the proof. We then construct the microlocal partition of unity whose properties are the central ingredient in the proof. Finally we prove successively the overall regularity of the trace of the wave operator, the smoothness away from lengths of diffractive geodesics, and the regularity away from lengths of geometric geodesics.

The strategy of the proof is an imitation of the following non-constructive proof of the Poisson relation for a compact boundaryless manifold $X$. Let $1=$ $\sum A_{i}^{*} A_{i}+R$ be a microlocal partition of unity, with the $A_{i}$ 's having microsupport 
in very small sets in $S^{*} X$ and $R \in \Psi^{-\infty}(X)$. Then if we let $\equiv$ denote equivalence modulo $\mathcal{C}^{\infty}(\mathbb{R})$, we have

$$
\operatorname{Tr} D_{t}^{2 k} U(t) \equiv \operatorname{Tr} \sum A_{i}^{*} A_{i} D_{t}^{2 k} U(t)=\sum \operatorname{Tr} A_{i} D_{t}^{2 k} U(t) A_{i}^{*}=\sum \operatorname{Tr} A_{i} \Delta^{k} U(t) A_{i}^{*} .
$$

Let $I \subset \mathbb{R}_{+}$be a small interval containing no lengths of closed geodesics. Provided the microlocal partition of unity is sufficiently fine, there are no bicharacteristics of length $t \in I$ with both endpoints in $\mathrm{WF}^{\prime} A_{i}$. Hence by Hörmander's propagation theorem, each term $A_{i} \Delta^{k} U(t) A_{i}^{*}$ maps distributions to smooth functions, therefore is of trace class. Hence the wave trace is smooth on $I$. Note that to make this argument rigorous, we should actually approximate the $A_{i}$ by smoothing operators in order to justify the formal manipulations in (4).

The simple approach sketched above requires modification on a conic manifold. First, owing to the global nature of the "indicial operator" at the boundary (one of the obstructions to compactness for operators in Melrose's b-calculus), we must settle for a partition of unity of the form $\sum_{j} \psi_{j}^{2}+\sum_{i} A_{i}^{*} A_{i}$ where the $\psi_{j}$ 's are smooth cutoff functions localizing at different boundary components and the $A_{i}$ 's have microsupport over a relatively compact subset of $X^{\circ}$. Furthermore, we only have a solid understanding of propagation of singularities for a short time, in which singularities cannot repeatedly interact with $\partial X$. Hence we will decompose $t=t_{0}+\ldots t_{L}$ with each term small, and write

$$
\begin{aligned}
& \operatorname{Tr} U(t) \equiv \\
& \operatorname{Tr}\left(\sum_{j} \psi_{j}^{2}+\sum_{i} A_{i}^{*} A_{i}\right) U\left(t_{0}\right)\left(\sum_{j} \psi_{j}^{2}+\sum_{i} A_{i}^{*} A_{i}\right) U\left(t_{1}\right) \ldots\left(\sum_{j} \psi_{j}^{2}+\sum_{i} A_{i}^{*} A_{i}\right) U\left(t_{L}\right) .
\end{aligned}
$$

We rewrite this sum as a single sum over terms of the form

$$
\operatorname{Tr}\left(B_{i_{0}}^{*} B_{i_{0}}\right) U\left(t_{0}\right)\left(B_{i_{1}}^{*} B_{i_{1}}\right) U\left(t_{1}\right) \ldots\left(B_{i_{L}}^{*} B_{i_{L}}\right) U\left(t_{L}\right)
$$

where each $B_{i}$ is either one of the $\psi_{j}$ 's or one of the $A_{i}$ 's. The strategy for the part of the theorem dealing with diffractive geodesics is to show that if the partition of unity is taken sufficiently fine, one factor in each summand of the form $B_{i_{j}} U\left(t_{j}\right) B_{i_{j+1}}^{*}$ (possibly after cyclic permutation), is smoothing. This is based on the absence of closed diffractive geodesics of length $t$, and on the principle that if there are no closed geodesics of length $t$ then there are no closed "broken" geodesics of such length which are repeatedly allowed to propagate for time $t_{j}$ and then jump arbitrarily within $\mathrm{WF}^{\prime} B_{i_{j}}$.

The fact that our partition of unity fails to localize over the boundary is of no import in dealing with diffractive geodesics which may, after all, jump freely from one point over the boundary to another in the same component. Difficulties arise, however, in dealing with geometric geodesics. In particular, the strategy outlined above does not work in the geometric case, and instead of considering terms of the form $B_{i_{j}} U\left(t_{j}\right) B_{i_{j+1}}^{*}$, if one of the $B$ 's is a localizer near a boundary component we may need to consider more complicated factors 
$B_{i_{j}} U\left(t_{j}\right) B_{i_{j+1}}^{*} B_{i_{j+1}} U\left(t_{j+1}\right) B_{i_{j+2}}^{*}$ so as to be considering interior-to-interior propagation of singularities. This situation requires the consideration of a number of cases, involving various possibilities for existence of geodesics from $\mathrm{WF}^{\prime} B_{i_{j+2}}$ to $\mathrm{WF}^{\prime} B_{i_{j+1}}$ and thence to $\mathrm{WF}^{\prime} B_{i_{j}}$.

4.1. Microlocal partition of unity. The reader may find it helpful to refer back and forth between this section and those following in which its results are employed to prove the theorem.

For $j=1, \ldots, K$, let $U_{j}$ denote a product neigborhood of $Y_{j}$ of the form $\left\{x<\epsilon_{j}\right\}$; let $\psi_{j} \in \mathcal{C}^{\infty}(X)$ denote a cutoff function supported in $U_{j}$, equal to 1 on a smaller neighborhood $U_{j}^{\prime}=\left\{x<\epsilon_{j}^{\prime}\right\}$ of $Y_{j}$. Let $\left\{V_{i}\right\}_{i=1}^{M}$ be an open cover of $S^{*} X \backslash \pi^{-1} \bigcup_{i} U_{i}^{\prime}$.

We now establish the existence of operators $A_{i} \in \Psi_{c}^{0}\left(V_{i}\right)$ such that

$$
R+\sum_{j=1}^{K} \psi_{j}^{2}+\sum_{i=1}^{M} A_{i}^{*} A_{i}=1
$$

with $R \in \Psi_{c}^{-\infty}\left(X^{\circ}\right)$ a compactly supported smoothing operator. $\left(\Psi_{c}^{0}\left(V_{i}\right)\right.$ denotes the space of pseudodifferential operators whose kernels have supports which project to compact subsets of $V_{i}$ on both left and right factors.) To do this note that using a symbolic construction in the ordinary pseudodifferential calculus, we can choose $D_{i} \in \Psi_{c}^{0}\left(V_{i}\right)$ such that $\mathrm{WF}^{\prime} \sum D_{i}^{*} D_{i}-1 \subset \bigcup U_{j}^{\prime}$. Then setting $\zeta=\left(1-\sum \psi_{j}^{2}\right)^{1 / 2}$, we may set $A_{i}=D_{i} \zeta$, hence $\sum A_{i}^{*} A_{i}-\zeta^{2} \in \Psi_{c}^{-\infty}\left(X^{\circ}\right)$, as desired.

Let $A_{i, \delta}, \delta \in[0,1]$, be a family in $\Psi^{-\infty}\left(X^{\circ}\right)$ converging strongly to $A_{i}$ as $\delta \downarrow 0$.

Let

$$
B_{i, \delta}=\left\{\begin{array}{l}
\psi_{i} \text { for } i=1, \ldots, K \\
A_{i-k, \delta} \text { for } i=K+1, \ldots K+M
\end{array}\right.
$$

let $B_{i}=B_{i, 0}$. Hence

$$
1=\sum B_{i}^{*} B_{i}+R
$$

Let $\bar{t} \in \mathbb{R}_{+}$be sufficiently small that $\left\{d\left(Y_{i}, \cdot\right)<\bar{t}\right\}$ is contained in a product neighborhood of $Y_{i}$ for all $i$, and such that $\bar{t} \ll d\left(Y_{i}, Y_{j}\right)$ for all $i, j$.

Suppose that we are given $T \in \mathbb{R}_{+} \backslash$ DIFF. Decompose $T=t_{0}+\cdots+t_{L}$ such that $\bar{t} / 4<t_{i}<\bar{t} / 2$ for all $i$ (we may decrease $\bar{t}$ if necessary).

In the statement of the following lemma and thenceforth, we always consider indices $i_{0}, \ldots, i_{L}$ up to cyclic shift, i.e. $i_{l}$ is always shorthand for $i_{l \bmod L+1}$.

Lemma 11. Given $T=t_{0}+\ldots t_{L} \in \mathbb{R}_{+} \backslash$ DIFF with each $t_{i} \in(\bar{t} / 4, \bar{t} / 2)$, we may choose the cover $\mathcal{C}=\left\{\pi^{-1} U_{i}\right\} \cup\left\{V_{i}\right\}$ of ${ }^{b} S^{*} X$ sufficiently fine that for any $i_{0}, \ldots, i_{L}$ there exist $l$ and an open interval $I \ni t_{i_{l}}$ such that there does not exist a lifted diffractive geodesic with length in I, ending in $\mathrm{WF}^{\prime} B_{i_{l}}$, and beginning in $\mathrm{WF}^{\prime} B_{i_{l+1}}$. 
Proof. Let $\mathcal{C}^{m}$ denote a sequence of finer covers $\left\{\pi^{-1} U_{i}^{m}\right\} \cup\left\{V_{i}^{m}\right\}$, and $B_{l}^{m}$ the corresponding operators in our microlocal partition of unity. If the conclusion of the lemma fails to holds then for each $m$ there exists an $(L+1)$-tuple of pairs of points $\left(p_{0}^{m}, q_{0}^{m}\right), \ldots,\left(p_{L}^{m}, q_{L}^{m}\right)$ such that $p_{l}^{m}, q_{l}^{m} \in \mathrm{WF}^{\prime} B_{i_{l}}$ and $t_{l}^{m} \rightarrow t_{l}$ such that $p_{l+1}^{m} \stackrel{D, t_{l}^{m}}{\sim} q_{l}^{m}$. As $m \rightarrow \infty$, i.e. as the partition is refined, we can then extract a subsequence such that $p_{l}^{m} \rightarrow \bar{p}_{l}$ and $q_{l}^{m} \rightarrow \bar{q}_{l}$. If either $\bar{p}_{l}$ or $\bar{q}_{l}$ is in ${ }^{b} S^{*} X^{\circ}$, then $\bar{p}_{l}=\bar{q}_{l}$, since the two points lie in a sequence of shrinking sets over $X^{\circ}$. If $\bar{p}_{l}$ or $\bar{q}_{l}$ is in ${ }^{b} S_{\partial X}^{*} X$ then the two points $\bar{p}_{l}$ and $\bar{q}_{l}$ must lie over the same component of $\partial X$. Thus by Proposition 4 , each $\bar{p}_{l+1}$ is connected to $\bar{p}_{l}$ by a diffractive geodesic of length $t_{l}$, hence these points must lie along a closed diffractive geodesic of length $T$ (recall that $l$ is considered modulo $L$ throughout), contradicting our assumptions.

In the case $T \in \mathbb{R}_{+} \backslash$ GEOM, we will need to impose a subtler set of conditions on our refined cover.

Lemma 12. Given $T=t_{0}+\ldots t_{L} \in \mathbb{R}_{+} \backslash$ GEOM with each $t_{i} \in(\bar{t} / 4, \bar{t} / 2)$, we may choose the cover $\left\{\pi^{-1} U_{i}\right\} \cup\left\{V_{i}\right\}$ of ${ }^{b} S^{*} X$ sufficiently fine that for any $i_{0}, \ldots, i_{L}$ there exist open intervals $I_{l} \ni t_{l}$ such that one of the following holds:

1. There exists $l$ such that $i_{l}$ and $i_{l+1}$ are both less than or equal to $K$ (i.e. are both $B_{i_{l}}$ and $B_{i_{l+1}}$ are $\psi$ 's), or

2. There exists $l$ such that $i_{l}$ and $i_{l+1}$ are both greater than $K$ and there does not exist a geometric geodesic with length in $I_{l}$, ending in $\mathrm{WF}^{\prime} A_{i_{l}-K}$, and beginning in $\mathrm{WF}^{\prime} A_{i_{l+1}-K}$, or

3. There exists $l$ such that $i_{l}, i_{l+2}>K$ and $i_{l+1} \leq K$ and there does not exist a geometric geodesic with length in $I_{l}+I_{l+1}$, ending in $\mathrm{WF}^{\prime} A_{i_{l}-K}$ and beginning in $\mathrm{WF}^{\prime} A_{i_{l+2}-K}$.

Proof. If the conclusion fails to hold, there must exist a sequence of shrinking covers $\mathcal{C}^{m}$ and shrinking intervals $I_{l}^{m} \ni t_{l}$ in each of which there exists a "word" $i_{0}^{m}, \ldots i_{L}^{m}$ and $\left(p_{l}^{m}, q_{l}^{m}\right) \in \mathrm{WF}^{\prime} B_{i_{l}}^{m}$ such that such that no two successive $i_{l}$ 's are less than or equal to $K$, and for all $l=1, \ldots L$, either

- If $i_{l}^{m}, i_{l+l}^{m}>K$, there exists a lifted geometric geodesic from $p_{i_{l+1}}^{m}$ to $q_{i_{l}}^{m}$ with length in $I_{l}^{m}$, or

- If $i_{l}^{m}>K$ and $i_{l+1}^{m} \leq K$, then $i_{l+2}^{m}>K$ and there exists a lifted geometric geodesic from $p_{i_{l+2}}^{m}$ to $q_{i_{l}}^{m}$ with length in $I_{l}^{m}+I_{l+1}^{m}$.

As $m \rightarrow \infty$, i.e. as the partition is refined, we may extract a subsequence such that $p_{l}^{m} \rightarrow \bar{p}_{l}$ and $q_{l}^{m} \rightarrow \bar{q}_{l}$. If eventually $i_{l}^{m}>K$, i.e. if $p_{l}^{m}$ and $q_{l}^{m}$ eventually lie in one of the interior sets $V_{k}^{m}$, then $\bar{p}_{l}=\bar{q}_{l}$. Hence if both $p_{l}^{m}$ and $p_{l+1}^{m}$ are such points then $\bar{p}_{l+1} \stackrel{G, t_{l}}{\sim} \bar{p}_{l}$ by Proposition 4 . If, on the other hand, we eventually have $i_{l}^{m}>K$ but $i_{l+1}^{m} \leq K$, then $i_{l+2}^{m}>K$, and we must have $\bar{p}_{l+2} \stackrel{G, t_{l}+t_{l+1}}{\sim} \bar{p}_{l}$ by Proposition 4 . Thus, by Proposition 4 , the points $\bar{p}_{l}$ must all lie along a geometric geodesic of length $T$, contradicting $T \notin$ GEOM. 
Lemma 13. By further refining the cover $V_{j}$ and shrinking the intervals $I_{j}$ we may assume in case 3 of Lemma 12 that either

i. There exist no diffractive geodesics with length in $I_{l}$ ending in $\mathrm{WF}^{\prime} A_{i_{l}-K}$, and beginning in $\pi^{-1} \operatorname{supp} \psi_{i_{l+1}}$, or

ii. There exist no diffractive geodesics with length in $I_{l+1}$ ending in $\pi^{-1} \operatorname{supp} \psi_{i_{l+1}}$ and beginning in $\mathrm{WF}^{\prime} A_{i_{l+2}-K}$, or

iii. Any diffractive geodesic of length $\mathcal{L} \in I_{l}+I_{l+1}$ beginning in $\mathrm{WF}^{\prime} A_{i_{l+2}-K}$ and ending in $\mathrm{WF}^{\prime} A_{i_{l}-K}$ must lie in $\pi^{-1} \operatorname{supp} \psi_{i_{l+1}}$ either for all $t \in I_{l+1}$ or for all $t \in \mathcal{L}-I_{l}$, or

iv. No diffractive geodesics with length in $I_{l}$ ending in $\mathrm{WF}^{\prime} A_{i_{l}-K}$ pass through $\partial X$, or

v. No diffractive geodesics with length in $I_{l+1}$ beginning in $\mathrm{WF}^{\prime} A_{i_{l+2}-K}$ pass through $\partial X$.

Proof. Since $\bar{t} \ll \min d\left(Y_{i}, Y_{j}\right)$ for any given values of $i_{l}, i_{l+2}$ there is at most one value of $i_{l+1}$ - call it $\bar{l}$-for which (i) and (ii) fail to hold (provided the intervals $I_{l}$ are chosen sufficiently small). If $I_{l}$ is a sufficiently small interval, the set $\bigcap_{t \in \bar{I}_{l}}\left\{p: q \stackrel{D, t}{\sim} p \Rightarrow q \in \pi^{-1} U_{\bar{\imath}}^{\prime}\right\}$ is an open neighborhood of the compact set $\bigcup_{t \in \bar{I}_{l}}\left\{p: q \stackrel{D, t}{\sim} p\right.$ for some $\left.q \in \pi^{-1} Y_{\bar{i}}\right\}$ (cf. Lemma 4.5 of [11]), hence we may refine the cover $\left\{V_{j}\right\}$ so that any $V_{j}$ intersecting the latter lies inside the former.

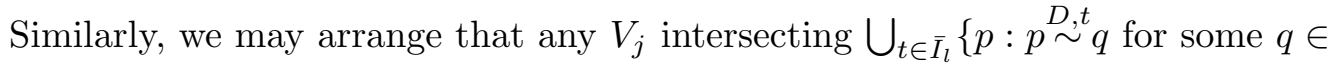
$\left.\pi^{-1} Y_{\bar{\imath}}\right\}$ lies inside $\bigcap_{t \in \bar{I}_{l}}\left\{p: p \stackrel{D, t}{\sim} q \Rightarrow q \in \pi^{-1} U_{\bar{\imath}}^{\prime}\right\}$.

Any diffractive geodesic with length in $I_{l}+I_{l+1}$ ending in $\mathrm{WF}^{\prime} A_{i_{l}-K}$, and beginning in $\mathrm{WF}^{\prime} A_{i_{l+2}-K}$ must pass through the boundary component over $Y_{\bar{\imath}}$, as there are no geometric geodesics connecting such points. Let $\gamma$ be such a geodesic (time-parametrized) and $\mathcal{L}$ its length. If $\gamma(t)$ passes through $Y_{\bar{\imath}}$ for some $t \in I_{l+1} \cup\left(\mathcal{L}-I_{l}\right)$ then by our refinement of the cover in the previous paragraph, $\gamma(t) \in \pi^{-1} U_{\bar{\imath}}^{\prime}$ for all $t$ in either $I_{l+1}$ or $\mathcal{L}-I_{l}$, hence case (iii) holds.

If, on the contrary, every geodesic $\gamma(t)$ with length in $I_{l}+I_{l+1}$ ending in $\mathrm{WF}^{\prime} A_{i_{l}-K}$, and beginning in $\mathrm{WF}^{\prime} A_{i_{l+2}-K}$ passes through $Y_{\bar{\imath}}$ at time $\tau \notin I_{l+1} \cup$ $\left(\mathcal{L}-I_{l}\right)$, we have two cases, $\tau \gtrless I_{l+1} \cup\left(\mathcal{L}-I_{l}\right)$. We may assume, by further refinement of the cover, that just one of these inequalities holds for all the aforementioned diffractive geodesics with a given $i_{l}, i_{l+2}$; indeed we may assume that the set of lengths of geodesics connecting a $V_{j}$ to a boundary component is a single interval. Say we are in the case $>$. Then all geodesics from $\mathrm{WF}^{\prime} A_{i_{l+2}-K}$ to $\pi^{-1} Y_{\bar{\imath}}$ have length greater than $I_{l+1}$, i.e. case (v) holds. The proof with sign $<$ is analogous: case (iv) holds.

We will need one further piece of geometric information about the partition of unity. 
Lemma 14. Let $\bar{t}$ be fixed as above. If the sets $U_{j} \supset Y_{j}$ are chosen sufficiently small, then there are no diffractive geodesics of length in $[\bar{t} / 4, \bar{t} / 2]$ connecting points in $U_{j}$ with points in $U_{k}$ for any $j, k$.

Proof. The result is clear for $j \neq k$, since $\bar{t}$ is less than the distance between boundary components. Thus we treat the case $j=k$. It suffices to show that for any $j$, there exists $\epsilon$ sufficiently small that any diffractive geodesic starting in the component of $\{x<\epsilon\}$ containing $Y_{j}$ must lie in $\{x>\epsilon\}$ for $t \in[\bar{t} / 4, \bar{t} / 2]$. Any diffractive geodesic passing through $Y_{j}$ is locally of the form $y=y_{0}, x=x_{0} \pm t$; along such a diffractive geodesic, $t=x \pm x_{0}$, hence either $x$ or $x_{0}$ must exceed $\bar{t} / 8$ when $t>\bar{t} / 4$. Hence it suffices, in considering such geodesics, merely to take $\epsilon=\bar{t} / 8$.

We now consider geodesics which do not pass through $Y_{j}$. We consider the unit speed geodesic flow to be the flow given by $(2)$ in ${ }^{b} T^{*} X$, inside $g=\left(\xi^{2}+\right.$ $h(x, y, \eta)) / x^{2}=1$. If $x_{0}<\epsilon$, then of course $\left|\xi_{0}\right|<\epsilon$. On the other hand $\dot{\xi}=\left(\xi^{2}+h\right) / x^{2}+\mathcal{O}\left(\eta^{2} / x\right) \geq 1 / 2$ if $\epsilon$ is sufficiently small. Hence $\xi(t) \geq \xi_{0}+t / 2 \geq$ $-\epsilon+t / 2$. Since $\dot{x}=\xi / x$, we obtain $x^{2} \geq t^{2} / 2-2 \epsilon t$, which is greater than $\epsilon^{2}$ for $t>\bar{t} / 4$ if $\epsilon$ is sufficiently small.

4.2. Overall regularity. We begin the proof of the theorem by establishing the overall regularity

$$
\operatorname{Tr} U(t) \in \mathcal{C}^{-n-0}(\mathbb{R}) .
$$

To do this, note that for $\epsilon>0$ and all $s \in \mathbb{R}$,

$$
\Theta_{-n-\epsilon} U(t): \mathcal{E}_{s} \rightarrow \mathcal{E}_{s+n+\epsilon},
$$

hence by Lemma $7, \Theta_{-n-\epsilon} U(t)$ is locally in $L^{\infty}\left(\mathbb{R} ; \mathcal{I}_{1}\left(\mathcal{E}_{s}\right)\right)$, i.e. $\operatorname{Tr} U(t)$ $\in \mathcal{C}^{-n-0}(\mathbb{R})$ as desired.

4.3. Diffractive lengths. We now show that $U(t) \in \mathcal{C}^{\infty}(\mathbb{R} \backslash D I F F)$. It suffices by selfadjointess of $\Delta$ to show smoothness in $\mathbb{R}_{+} \backslash$ DIFF.

We now choose the cover $\mathcal{C}$ as guaranteed by Lemma 11, and compute

$$
\begin{gathered}
\operatorname{Tr} D_{t}^{k(L+1)} U\left(t+t_{1}+\cdots+t_{n}\right)=\lim _{\delta \downarrow 0} \operatorname{Tr}\left(R+\sum B_{i, \delta}^{*} B_{i, \delta}\right) D_{t}^{k} U(t) \\
\cdot\left(R+\sum B_{i, \delta}^{*} B_{i, \delta}\right) D_{t}^{k} U\left(t_{1}\right)\left(R+\sum B_{i, \delta}^{*} B_{i, \delta}\right) \ldots\left(R+\sum B_{i, \delta}^{*} B_{i, \delta}\right) D_{t}^{k} U\left(t_{L}\right)
\end{gathered}
$$

in the sense of distributions (since $1-\sum B_{i, \delta}^{2}-R$ approaches zero in norm as a $\operatorname{map} \mathcal{D}_{s} \rightarrow \mathcal{D}_{s+\epsilon}$ for all $\epsilon>0$ ). Now expand out the above sum. By Lemma 11, each term of the form

$$
B_{i_{0}, \delta}^{*} B_{i_{0}, \delta} D_{t}^{k} U(t) B_{i_{1}, \delta}^{*} B_{i_{1}, \delta} \ldots B_{i_{L}, \delta}^{*} B_{i_{L}, \delta} D_{t}^{k} U\left(t_{L}\right)
$$

contains (after a possible cyclic permutation) a factor $B_{i_{l}, \delta} D_{t}^{k} U\left(t_{i_{l}}\right) B_{i_{l+1}, \delta}^{*}$ that, by Propositions 8 and 10, maps $\mathcal{E}_{-\infty} \rightarrow \mathcal{E}_{\infty}$ (uniformly in $\delta$ ), while the rest of the terms map $\mathcal{E}_{s} \rightarrow \mathcal{E}_{s-k}$ for all $s \in \mathbb{R}$. Hence the expression as a whole is of trace class, uniformly in $t$ near $t_{0}$ and as $\delta \downarrow 0$. Any term involving a 
factor of $R$ also has this property, since $R: \mathcal{E}_{-\infty} \rightarrow \mathcal{E}_{\infty}$. Thus, for all $k \in \mathbb{N}_{0}$, $D_{t}^{k(L+1)} U\left(t+t_{1}+\cdots+t_{L}\right) \in L^{\infty}\left(\mathbb{R} ; \mathcal{I}_{1}\left(\mathcal{E}_{s}\right)\right)$ near $t=t_{0}$, proving that

$$
U(t) \in \mathcal{C}^{\infty}(\mathbb{R} \backslash \mathrm{DIFF}) .
$$

4.4. Geometric lengths. It now remains to show

$$
U(t) \in \mathcal{C}^{-1-0}(\mathbb{R} \backslash \mathrm{GEOM}) .
$$

Let $T \in \mathbb{R}_{+} \backslash$ GEOM and decompose $T$ into a sum as before. We have for $\epsilon>0$,

$$
\begin{aligned}
\Theta_{-1-\epsilon} U\left(t+t_{1}+\cdots+t_{L}\right) & =\Theta_{-1-\epsilon} U(t) U\left(t_{1}\right) \ldots U\left(t_{L}\right) \\
= & \left(R+\sum B_{i, \delta}^{*} B_{i, \delta}\right) \Theta_{-1-\epsilon} U(t) \\
& \cdot\left(R+\sum B_{i, \delta}^{*} B_{i, \delta}\right) U\left(t_{1}\right) \\
& \ldots\left(R+\sum B_{i, \delta}^{*} B_{i, \delta}\right) U\left(t_{L}\right),
\end{aligned}
$$

For brevity's sake, let

$$
W_{j}= \begin{cases}U\left(t_{j}\right), & j \neq 0 \\ \Theta_{-1-\epsilon} U(t), & j=0 .\end{cases}
$$

Applying Lemma 12, we may refine the cover of ${ }^{b} S^{*} X$ sufficiently that any word

$$
B_{i_{0}}^{*} B_{i_{0}} W_{0} B_{i_{1}}^{*} B_{i_{1}} W_{1} B_{i_{2}}^{*} B_{i_{2}} \ldots B_{i_{L}}^{*} B_{i_{L}} W_{L},
$$

in (5) contains (modulo cyclic permutation) a factor of one of the following forms, corresponding to the various cases of the lemma:

1. $\psi_{i_{l}} W_{l} \psi_{i_{l+1}}$, or

2. $A_{i_{l}-K} W_{l} A_{i_{l+1}-K}^{*}$ where there does not exist a geometric geodesic with length in $I_{l}$ ending in $\mathrm{WF}^{\prime} A_{i_{l}-K}$, and beginning in $\mathrm{WF}^{\prime} A_{i_{l+1}-K}$, or

3. $A_{i_{l}-K} W_{l} \psi_{i_{l+1}}^{2} W_{l+1} A_{i_{l+2}-K}^{*}$ where there does not exist a geometric geodesic with length in $I_{l}+I_{l+1}$ ending in $\mathrm{WF}^{\prime} A_{i_{l}-K}$, and beginning in $\mathrm{WF}^{\prime} A_{i_{l+2}-K}$

The term in case 1 above is certainly of trace class, since by Lemma 14 there are no diffractive geodesics (let alone geometric ones) connecting points in $\psi_{i_{l}}$ and $\psi_{i_{l+1}}$ and having length in $[\bar{t} / 4, \bar{t} / 2]$. Hence any term

$$
B_{i_{0}, \delta}^{*} B_{i_{0}, \delta} \Theta_{-1-\epsilon} U(t) B_{i_{1}, \delta}^{*} B_{i_{1}, \delta} U\left(t_{1}\right) B_{i_{2}, \delta}^{*} B_{i_{2}, \delta} \ldots B_{i_{L}, \delta}^{*} B_{i_{L}, \delta} U\left(t_{L}\right),
$$

containing such a factor is uniformly of trace class in $t$ near $t_{0}$ and as $\delta \downarrow 0$.

Proposition 9 shows that a term of the type in case 2,

$$
A_{i_{l}-K} U(s) A_{i_{l+1}-K}^{*},
$$

where there are no geometric geodesics with lengths in $I_{l}$ between the microsupports of the outer terms, is in fact a system of Fourier integral operators in $x$ of order $+\epsilon$ with values in $\Psi^{-\infty}\left(Y_{j}\right)$ for some $j$, uniformly in $s \in I_{l}$. (We 
are assuming for the moment that $l \neq 0$.) Now let $D \in \Psi^{-1-\epsilon}\left(X^{\circ}\right)$ be compactly supported and elliptic on $\mathrm{WF}^{\prime} A_{i_{l}-K}$; let $E \in \Psi^{+1+\epsilon}\left(X^{\circ}\right)$ be a compactly supported microlocal parametrix for $D$, hence $\mathrm{WF}^{\prime} E D \cap \mathrm{WF}^{\prime} A_{i_{l}-K}=\emptyset$. Then

$$
A_{i_{l}-K} U\left(t_{l}\right) A_{i_{l+1}-K}^{*}=E D A_{i_{l}-K} U\left(t_{l}\right) A_{i_{l+1}-K}^{*}+S
$$

where $S$ is a compactly supported smoothing operator on $X^{\circ}$, hence does not contribute to singularities of the trace. The term

$$
D A_{i_{l}-K, \delta} U\left(t_{l}\right) A_{i_{l+1}-K, \delta}^{*}
$$

is then of trace class, uniformly in $\delta$; hence the whole term

$$
\operatorname{Tr} B_{i_{0}, \delta}^{*} B_{i_{0}, \delta} \Theta_{-1-\epsilon} U(t) B_{i_{1}, \delta}^{*} B_{i_{1}, \delta} U\left(t_{1}\right) B_{i_{2}, \delta}^{*} B_{i_{2}, \delta} \ldots B_{i_{L}, \delta}^{*} B_{i_{L}, \delta} U\left(t_{L}\right)
$$

can be written, modulo an error term mapping $\mathcal{E}_{-\infty} \rightarrow \mathcal{E}_{\infty}$, as a product of factors preserving $\mathcal{E}_{s}$ for all $s$, times a factor $\Theta_{-1-\epsilon} U(t)$ which maps $\mathcal{E}_{s} \rightarrow \mathcal{E}_{s+1+\epsilon}$ for all $s \in \mathbb{R}$ and $\epsilon>0$, times a factor $E$ which maps $\mathcal{E}_{s} \rightarrow \mathcal{E}_{s-1-\epsilon}$, times a factor

$$
D A_{i_{l}-K, \delta} U\left(t_{l}\right) A_{i_{l+1}-K, \delta}^{*} \in \mathcal{I}_{1}\left(\mathcal{E}_{s}\right),
$$

uniformly in $\delta$. Hence this term has a trace uniformly bounded as $\delta \downarrow 0$. The terms involving the smoothing error $R$ do not contribute, as before. The separate case $l=0$ works similarly.

In the subcases (i) and (ii) of case (3) (described in Lemma 13) the corresponding term in the decomposition of $U(t)$ again maps $\mathcal{E}_{-\infty} \rightarrow \mathcal{E}_{\infty}$, hence does not contribute to singularities of the trace. To deal with subcase (iii), we decompose

$$
\begin{aligned}
& A_{i_{l}-K} U\left(t_{l}\right) \psi_{i_{l+1}}^{2} U\left(t_{l+1}\right) A_{i_{l+2}-K}^{*} \\
& \quad=A_{i_{l}-K} U\left(t_{l}+t_{l+1}\right) A_{i_{l+2}-K}^{*}+A_{i_{l}-K} U\left(t_{l}\right)\left(1-\psi_{i_{l+1}}^{2}\right) U\left(t_{l+1}\right) A_{i_{l+2}-K}^{*} .
\end{aligned}
$$

The second term maps $\mathcal{E}_{-\infty} \rightarrow \mathcal{E}_{\infty}$, while the first can be dealt with just as the similar term in case (2). For case (iv), note that by Egorov's theorem, there exists $\tilde{A} \in \Psi_{c}^{0}\left(X^{\circ}\right)$ with microsupport in the geodesic flowout for time $-t_{l}$ of $\mathrm{WF}^{\prime} A_{i_{l}-K}$ such that

$$
A_{i_{l}-K} U\left(t_{l}\right) \psi_{i_{l+1}}^{2} U\left(t_{l+1}\right) A_{i_{l+2}-K}^{*}=U\left(t_{l}\right) \tilde{A} U\left(t_{l+1}\right) A_{i_{l+2}-K}^{*} .
$$

Now by assumption, there are no geometric geodesics of length in $I_{l+1}$ beginning in $\mathrm{WF}^{\prime} A_{i_{l+2}-K}$ and ending in $\mathrm{WF}^{\prime} \tilde{A}$, hence we may proceed as in case (2), since by [7], the term $\tilde{A} U\left(t_{l+1}\right) A_{i_{l+2}-K}^{*}$ is a Fourier integral operator in $x$ of order $+\epsilon$ with values in $\Psi^{-\infty}\left(Y_{j}\right)$ for some $j$. Case (v) of Lemma 13 is analogous.

\section{Acknowledgements}

The author is grateful to Richard Melrose for helpful discussions and to an anonymous referee for substantial improvements to the exposition. This research was supported in part by NSF grant DMS-0100501. 


\section{References}

[1] J. Chazarain, Formule de Poisson pour les variétés riemanniennes, Invent. Math. 24 (1974), 65-82.

[2] J. Cheeger, M. Taylor, On the diffraction of waves by conical singularities. I, Comm. Pure Appl. Math. 35 (1982), 275-331.

[3] - On the diffraction of waves by conical singularities. II, Comm. Pure Appl. Math. 35 (1982), 487-529.

[4] J. J. Duistermaat, V.W. Guillemin, The spectrum of positive elliptic operators and periodic geodesics, Invent. Math. 29 (1975), 39-79.

[5] P. Gérard, G. Lebeau, Diffusion d'une onde par un coin, J. Amer. Math. Soc. 6 (1993), 341-424.

[6] L. Hörmander, On the existence and the regularity of solutions of linear pseudodifferential equations, Enseignement Math. (2) 17 (1971), 99-163.

[7] R. B. Melrose, J. Wunsch, Propagation of singularities for the wave equation on conic manifolds, preprint.

[8] R. B. Melrose, Transformation of boundary problems, Acta Math. 147 (1981), 149-236.

[9] _ The Atiyah-Patodi-Singer index theorem, Research Notes in Mathematics, 4. A K Peters, Ltd., Wellesley, MA, 1993.

[10] M. Taylor, Partial differential equations. III, Corrected reprint of the 1996 original. Applied Mathematical Sciences, 117. Springer-Verlag, New York, 1997.

[11] J. Wunsch, The trace of the generalized harmonic oscillator, Ann. Inst. Fourier (Grenoble) 49 (1999), 351-373.

Department of Mathematics, Northwestern University, 2033 Sheridan Road, EvANSTON, IL 60208-2730, U.S.A.

E-mail address: jwunsch@math.northwestern.edu 